\title{
Genes and the eye
}

Mariya Moosajee MB BSc

J R Soc Med 2005;98:206-207

For those who see genetics as the potential answer to many ills, these are exciting times. A vast flood of information from the Human Genome Project, completed just two years ago, ${ }^{1}$ is already elucidating the replication, recombination and segregation of chromosomes. For gene therapies, however, we require knowledge of products and their functions, ${ }^{2}$ and the next stage is to examine the transcriptome, the metabolome and the proteome. The Human Phenome Project, ${ }^{3}$ by determining the relations between genotypes and phenotypes, should move us towards an era of individualized prevention and treatment. ${ }^{4}$ An organ for which this approach holds particular promise, in 2005, is the eye.

\section{The eye}

Nearly a century has passed since an ocular phenotype, that of colour blindness, was assigned to the X chromosome. ${ }^{5}$ In the interim, numerous ocular disease loci have been mapped and several causal genes have been identified. Some of the single-gene disorders, such as Doyne honeycomb retinal dystrophy, are non-life-threatening and can be found in several generations of an affected family. ${ }^{6}$ By contrast, agerelated macular degeneration and diabetic retinopathy are influenced by a complex mix of genetic and environmental factors that is hard to disentangle. ${ }^{7}$ Of the four thousand or more human genetic disorders that have been catalogued, ${ }^{8}$ at least $10 \%$ directly or indirectly affect the retina. Worldwide, about 1 in every 3000 individuals has a defect that will lead to retinal degeneration and subsequent partial or total blindness. Attempts to construct a reference transcriptome of the adult mammalian retina and retinal pigment epithelium - the retinome- have now begun. When completed, it will serve as a reference map to aid in identifying the genes responsible for retinal dystrophies, both monogenic and polygenic. In the future, registers of retinal gene mutations will be linked to the genotyping service, allowing screening for preventable or treatable disorders.

As I write in early 2005, three new approaches to treatment are nearing application-gene therapy, cell

Mariya Moosajee, MB BSc, is a senior house officer pursuing a career in ophthalmology. She is a member of the RSM's Sections of Medical Genetics and Ophthalmology.

Accident and Emergency Department, University College Hospital, London WC1E 6AU, UK

E-mail: mariya_arts@hotmail.com transplantation and the use of growth factors to delay cell death. ${ }^{9}$ For any such method to succeed, it has to be matched to the disease mechanism, ${ }^{10}$ and in the case of retinitis pigmentosa this is a progressive apoptotic loss of cells within the retina. Dominant retinitis pigmentosa is broadly divided into type I, diffuse, and type II, regional. Investigations have shown that in type I there is widespread loss of scotopic function (mediated by rod cells) with relative preservation of cones and an unexpectedly high level of residual rhodopsin. Here, gene therapy might be the best way to secure recovery of function. In type II the loss of rod and cone mediated sensitivities is patchy but about equal, and residual rhodopsin concentrations are proportionally low-thus, cell transplantation might be preferable, to obtain regeneration of both types of photoreceptor cell. ${ }^{10}$

The eye does have the great advantage of accessibility for local treatments, with their lesser risk of systemic effects. One of the most promising approaches, successful in rodent and $\operatorname{dog}$ models, is the use of a recombinant adenoassociated virus (rAAV) as gene delivery vector. The Briard dog carries the RPE65 null mutation defect present in 10\% of human beings with the childhood-onset retinal dystrophy Leber's congenital amaurosis. These animals do not regenerate adequate amounts of photopigment and in consequence have poor vision and show depressed adaptation to light and dark on the electroretinogram. ${ }^{11}$ When Briard dogs were transfected with a canine RPE65 gene by means of a rAAV vector, their responses on the electroretinogram were much improved. ${ }^{12}$ The downside was that uveitis developed in three-quarters of the treated eyes, and this adverse effect needs to be explained and eliminated before trials can be conducted in man.

Stem cell transplantation has likewise given promising results in animals. For example, one research group has recorded cone-cell rescue in two murine models of retinitis pigmentosa, with use of bone-marrow-derived lineagenegative stem cells from both genetically defective and wild type mice. ${ }^{13}$ Incorporation of these stem cells into retinal blood vessels led to stabilization and correction of the vascular degeneration that usually occurs with photoreceptor loss. In this experiment central cone vision was preserved without the adverse effects encountered with viral-vector gene therapy; rod cells were not rescued. These findings suggest that transplantation of a patient's 
own (genetically defective) bone marrow cells may offer cone neuroprotection via the expression of trophic factors so far unidentified.

\section{The next fifty years}

The above examples illustrate the potential for therapeutic application of genetic knowledge in eye disorders. These are early days, and when the time comes for trials in human beings we shall need internationally agreed protocols for end points such as electroretinography, imaging and psychophysical testing. ${ }^{10}$

Although the science of inheritance is beset with ethical dilemmas, its onward march is certain. Some of the present anxieties - for example concerning designer babies or the possible behaviour of insurance companies - might be dispelled by stronger efforts to educate the health professions and the public. By the time you see this article, dear reader in 2055, I suspect that genetics will long since have become integrated into clinical medicine, offering a means to diagnosis, treatment and prevention of a multitude of diseases. Genetics is our vision for the future.

\section{REFERENCES}

1 Austin CP. The impact of the completed human genome sequence on the development of novel therapeutics for human disease. Ann Rev Med 2004;55:1-13
2 Nebert DW, Vesell ES. Advances in pharmacogenomics and individualising drug therapy: exciting challenges that lie ahead. Euro J Pharmacol 2004;500:267-80

3 Freimer N, Sabatti C. The Human Phenome Project. $N$ Genet 2003;34:15-21

4 Scriver CR. After the genome- the phenome? J Inherit Metab Dis 2004;27:305-17

5 Wilson EB. The sex chromosome. Arch Mikrusk Anal Entwicklungsmech 1911;77:249-71

6 Blackburn J, Tarttelin EE, Gregory-Evans CY, Moosajee M, GregoryEvans K. Transcriptional regulation and expression of the dominant drusen gene FBLN3 (EFEMP1) in mammalian retina. Invest Ophthalmol Vis Sci 2003;44:4613-21

7 Klein ML, Francis PJ. Genetic of age-related macular degeneration. Ophthalmol Clin N Am 2003;16:567-74

8 McKusick VA. Mendelian Inheritance in Man. Baltimore: Johns Hopkins University Press, 1992

9 Schulz HL, Goetz T, Kaschkoetoe J, Weber BHF. The retinomedefining a reference transcriptome of the adult mammalian retina/ retinal pigment epithelium. BMC Genomics 2004;5:50-61

10 Bird AC. What should a clinician know to be prepared for the advent of treatment of retinal dystrophies? Novartis Found Symp 2004;255:85-94

11 Rolling F. Recombinant AAV-mediated gene transfer to the retina: gene therapy perspectives. Gene Therapy 2004;11:S26-32

12 Narfstrom K, Katz ML, Bragadothir R, et al. Functional and structural recovery of the retina after gene therapy in the RPE65 null mutation dog. Invest Ophthalmol Vis Sci 2003;44:1663-72

13 Otani A, Dorrell MI, Kinder K, et al. Rescue of retinal degeneration by intravitreally injected adult bone marrow-derived lineage-negative hematopoietic stem cells. J Clin Invest 2004;114:765-74 